\title{
BMJ Open Association of hepatitis B surface antigen seropositivity and hepatitis $B$ surface antibody seropositivity with diabetes: a cross-sectional study based on two Chinese populations in Guangdong, China
}

\author{
Yuan Liu, ${ }^{1}$ Chaoqiang Jiang, ${ }^{2}$ Yuantao Hao (D) , ${ }^{1}$ Lin Xu, ${ }^{1,3}$ Weisen Zhang, ${ }^{2}$ \\ Ya Li Jin, ${ }^{2}$ Tong Zhu, ${ }^{2}$ Tai Hing Lam ${ }^{2,3}$
}

To cite: Liu Y, Jiang C, Hao Y, et al. Association of hepatitis B surface antigen seropositivity and hepatitis B surface antibody seropositivity with diabetes: a cross-sectional study based on two Chinese populations in Guangdong, China. BMJ Open 2020;10:e028968. doi:10.1136/ bmjopen-2019-028968

- Prepublication history and additional material for this paper are available online. To view these files, please visit the journal online (http://dx.doi. org/10.1136/bmjopen-2019028968).

Received 05 January 2019 Revised 10 December 2019 Accepted 22 January 2020

Check for updates

(C) Author(s) (or their employer(s)) 2020. Re-use permitted under CC BY-NC. No commercial re-use. See rights and permissions. Published by BMJ.

${ }^{1}$ School of Public Health, Sun Yat-Sen University, Guangzhou, Guangdong, China

${ }^{2}$ Guangzhou No.12 Hospital, Guangzhou, Guangdong, China ${ }^{3}$ School of Public Health, University of Hong Kong, Hong Kong, Hong Kong

Correspondence to

Yuantao Hao;

haoyt@mail.sysu.edu.cn

\section{ABSTRACT}

Objectives To examine whether hepatitis B surface antigen ( $\mathrm{HBsAg}$ ) seropositivity and hepatitis B surface antibody (HBsAb) seropositivity were associated with the presence of diabetes in two population-based studies in southern China, the Guangzhou Biobank Cohort Study (GBCS) and the Major Infectious Disease Prevention and Control (MIDPC) study.

Design A cross-sectional study.

Setting The GBCS was conducted among a community social and welfare organisation with branches in all 10 districts of Guangzhou. The MIDPC was conducted among the community residents in two districts of Guangzhou and three districts of Zhongshan.

Participants 4947 participants from the GBCS and 4357 participants from the MIDPC were included in this study. Primary and secondary outcome measures Type 2 diabetes was the main study outcome, which was diagnosed by fasting blood glucose $\geq 7.0 \mathrm{mmol} / \mathrm{L}$, and/or self-reported history of diabetes.

Results After adjusting for age, sex, education, occupation, smoking status, alcohol use, physical activity and body mass index, we found no association of $\mathrm{HBsAg}$ seropositivity in GBCS or MIDPC (OR=1.12, $95 \% \mathrm{Cl} 0.74$ to 1.69 , and $\mathrm{OR}=0.83,95 \% \mathrm{Cl} 0.59$ to 1.17 , respectively), and $\mathrm{HBsAb}$ seropositivity $(\mathrm{OR}=0.85,95 \% \mathrm{Cl} 0.65$ to $1.12, \mathrm{OR}=1.00,95 \% \mathrm{Cl} 0.86$ to 1.16 , respectively) with the presence of diabetes. Null associations were found for analysis pooling GBCS and MIDPC data after similar adjustment. The adjusted OR for the associations of $\mathrm{HBsAg}$ seropositivity and $\mathrm{HBsAb}$ seropositivity with the presence of diabetes in the pooled sample was $0.91(95 \% \mathrm{Cl} 0.70$ to $1.19)$ and 0.98 (95\% Cl 0.86 to 1.12), respectively. Conclusions Taking advantage of data from two large cross-sectional studies, we found no association of serological status of $\mathrm{HBsAg}$ and $\mathrm{HBsAb}$ with the presence of diabetes or glucose measures.

\section{INTRODUCTION}

Hepatitis B virus (HBV) infection is a major infectious disease in the world, especially in
Strengths and limitations of this study

- This is the first population-based study examining the association between hepatitis B surface antibody seropositivity and diabetes in China.

- The current study used data from two large population-based studies, the Guangzhou Biobank Cohort Study (GBCS) and the Major Infectious Disease Prevention and Control (MIDPC) study, and adjusted for multiple potential confounders, which might have increased the internal validity of the study.

- Due to the funding constraints, only $27.3 \%$ of participants in GBCS and $2.6 \%$ in MIDPC had data on both fasting glucose andhepatitis $B$ virus serological tests and were included in the data analysis, which might introduce selection bias and influence the generalisability of study results.

- There is a possibility of volunteer bias, because all residents were invited for free health check in the MIDPC, individuals who were more health conscious tended to join in the study.

China. In 1992, the prevalence of HBV infection indicated by hepatitis B surface antigen (HBsAg) positive in general Chinese population (aged 1-59 years) was 9.75\%.' Although the nationwide $\mathrm{HBV}$ vaccination programme for newborn babies was launched since 1992, the prevalence of $\mathrm{HBV}$ infection remained high (about $7.18 \%$ in 2006). ${ }^{2}$ A recent study showed that the average prevalence of HBV infection in the general Chinese population aged 1-59 years from 2007 to 2016 was $5.7 \%$. $^{3}$

Diabetes is a major public health problem globally, especially in China. A nationwide survey in 2007 and another large survey including participants from 31 provinces of 
China in 2010 showed that the diabetes prevalence was about $10 \%$ (ranged from $9.7 \%$ to $11.6 \%$ ). ${ }^{45}$ Such a high prevalence of diabetes in China imposes a very heavy burden on population health service as well as social and economic development. ${ }^{6}$

As HBV infection leads to poorer liver function, ${ }^{7}$ and the latter was associated with a higher risk of diabetes, ${ }^{89}$ many studies explored the association between HBV infection and diabetes, but the results were largely inconsistent in terms of the direction and the magnitude. ${ }^{10-23}$ Taking advantage of data from two population-based studies in Southern China (the Guangzhou Biobank Cohort Study (GBCS) and the Major Infectious Disease Prevention and Control (MIDPC) project), we examined whether HBsAg seropositivity and hepatitis B surface antibody (HBsAb) seropositivity were associated with the presence of diabetes in Chinese.

\section{METHODS}

\section{Study design}

This is a cross-sectional study using data from two large population-based studies in southern China, the GBCS and the MIDPC study.

\section{Data sources}

Guangzhou Biobank Cohort Study (GBCS)

The GBCS is a three-way collaboration among Guangzhou 12th Hospital and the Universities of Hong Kong and Birmingham, UK. Details of this study have been reported elsewhere. ${ }^{24}$ Briefly, participants were recruited from the Guangzhou Health and Happiness Association for the Respectable Elders (GHHARE), which is a community social and welfare organisation unofficially aligned with municipal government. Membership of the GHHARE is open to Guangzhou residents aged 50 years or above for a nominal fee of 4 RMB (equal to about 50 US cents) per month. GHHARE included about $7 \%$ of Guangzhou residents in this age group, with branches in all 10 districts of Guangzhou, the capital city of Guangdong province in southern China.

Information of demographic characteristics, environmental exposure, lifestyle, family and personal medical history was collected using a computer-assisted questionnaire-based face-to-face interview. Physical examination was conducted for anthropometric indices (weight, height, waist and hip circumference), and blood pressure, fasting plasma glucose, lipids, inflammatory markers, renal function and liver function were measured. In GBCS, all research staff received unified training before conducting the study. All participants gave written, informed consent before participation for free.

\section{MIDPC project}

The MIDPC project is a multicentre collaborative study among the National Science and Technology Major Special Office, Guangdong Provincial Local Government and Health and Family Planning Commission, Sun YatSen University, the third Affiliated Hospital of Sun YatSen University and the Centers for Disease Control and Prevention in Zhongshan and in Guangzhou. Free community-wide health examination and questionnaire survey were conducted for residents from two districts (Yuexiu and Liwan) in Guangzhou and three districts (Huoju, Guzhen and Xiaolan) in Zhongshan. All participants provided written informed consent before participation. From January 2014 to December 2015, 167105 participants were recruited in this study. A face-to-face questionnaire-based interview was conducted to collect information on demographic characteristics (age, sex, occupation, marital status, nationality and education level), lifestyle (alcohol use, smoking status and physical activity) and medical history (personal history of HBV infection, diabetes and hepatitis B vaccination). Information of anthropometric indices (weight, height and waist and hip circumference) and blood pressure was collected by physical examination. Clinical parameters including fasting plasma glucose, lipids, inflammatory markers and markers of liver and renal function were measured. In the MIDPC, all research staff also received rigorous training before the study. The biological samples were subject to centralised testing, using unified testing methods, experimental testing procedures and laboratory results judgement standards.

\section{Study sample}

In GBCS, 30430 Chinese aged 50 years or above participated in the baseline examination from 2003 to January 2008, and of them, 18105 returned for the first repeated examination from March 2008 to December 2012. After excluding participants with anti-HCV positivity, selfreported positive medical history of diabetes and participants without information of serological markers of hepatitis B and fasting/2 hour postload glucose, 4947 participants with all variables of interest were included in the current study. In MIDPC, after excluding participants aged 18 years or younger and without testing of hepatitis $\mathrm{B}$ infectious status and fasting glucose, 4357 participants were included in the current analysis. Also, to fully make use of the data, we examined the association of serological status of HBsAg and HBsAb with diabetes in the pooled data of GBCS and MIDPC.

\section{Study outcome}

Type 2 diabetes was the major study outcome in the present study, which was diagnosed by fasting blood glucose $\geq 7.0 \mathrm{mmol} / \mathrm{L}$, and/or self-reported history of diabetes. In GBCS, glycosylated haemoglobin A1c (HbAlc) and 2 hour postload glucose were also assessed. Both fasting and 2 hour postload glucose was measured in the laboratory of Guangzhou Number 12 Hospital using Roche COBAS C311 Chemistry Analyzer (Germany). In the MIDPC, fasting glucose was measured in the laboratory of Daan Gene Company of the Sun Yat-Sen University, Guangzhou, China. 
Table 1 Demographic characteristics and clinical parameters by HBsAg serological status (HBsAg positive vs negative) in GBCS and MIDPC

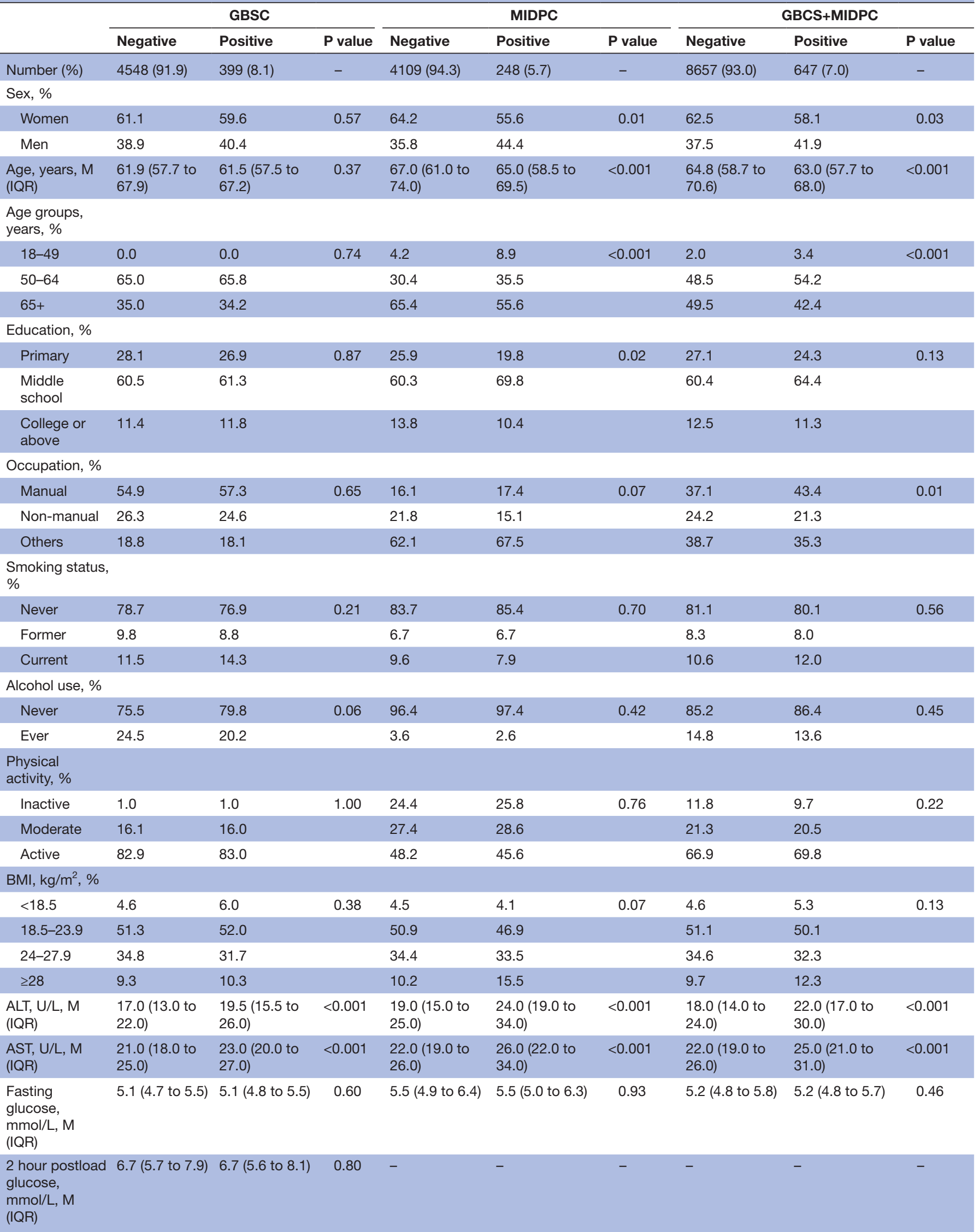


Table 1 Continued

\begin{tabular}{|c|c|c|c|c|c|c|c|c|c|}
\hline & \multicolumn{3}{|c|}{ GBSC } & \multicolumn{3}{|c|}{ MIDPC } & \multicolumn{3}{|c|}{ GBCS+MIDPC } \\
\hline & Negative & Positive & $\overline{P \text { value }}$ & Negative & Positive & $P$ value & Negative & Positive & $P$ value \\
\hline $\begin{array}{l}\text { HbA1c, \%, M } \\
\text { (IQR) }\end{array}$ & 5.9 (5.6 to 6.1$)$ & 5.9 (5.6 to 6.1$)$ & 0.80 & - & - & - & - & - & - \\
\hline Diabetes, \% & 6.5 & 6.8 & 0.83 & 30.1 & 28.6 & 0.62 & 17.7 & 15.2 & 0.10 \\
\hline
\end{tabular}

ALT, alanine transaminase; AST, aspartate transaminase; BMI, body mass index; GBCS, Guangzhou Biobank Cohort Study; HbA1c, glycated haemoglobin A1c; HBsAg, hepatitis B surface antigen; M, median; MIDPC, Major Infectious Disease Prevention and Control.

\section{Exposure}

In GBCS, serological testing for HBsAg and HBsAb was done using enzyme-linked immunoassay in the laboratory of Molecular Epidemiology Research Center of the Guangzhou Number 12 Hospital. Enzyme-linked immunoassay was also used in MIDPC for HBsAg and HBsAb testing in the laboratory of Daan Gene Company of the Sun Yat-Sen University. The presence of HBsAg was used as an indicator of $\mathrm{HBV}$ infection. ${ }^{25}$

\section{Patient and public involvement}

Patients and the public were not involved in this study.

\section{Statistical analysis}

Data with normal distribution data were described by mean and SD, and data with non-normal distribution were described by median and IQR. T-test and MannWhitney $\mathrm{U}$ test were used to examine the differences of continuous variables, and $\chi 2$ test was used for categorical variables. Logistic regression was used to examine the association of HBsAg and HBsAb serological status with the presence of diabetes, and linear regression was used to examine their association with the glycaemic measures. In the multivariable linear and logistic regression, potential confounders considered included age, sex, education, occupation, physical activity, smoking status, alcohol use and BMI. Moreover, we also examined whether the association of HBsAg and HBsAb serological status with diabetes varied by study sample source (ie, GBCS and MIDPC). As no evidence for the interaction with study sample source was found (all $\mathrm{p}>0.05$ ), additional analysis pooling two samples was done with adjustment for study sample source. All data analysis was conducted in STATA V.14.0. A two-sided p-value $\leq 0.05$ was considered statistically significant.

\section{RESULTS}

Characteristics of 9304 participants from two studies by HBsAg serological status were shown in table 1 . The seropositive rate of HBsAg was $8.1 \%(n=399)$ in GBCS and $5.7 \%(\mathrm{n}=248)$ in MIDPC. In GBCS, median (IQR) of age of HBsAg seropositive group was $61.5(57.5,67.2)$ years, while the HBsAg seronegative group was $61.9(57.7,67.9)$ years. No significant differences were found for age, sex, education, occupation, smoking status, alcohol use, physical activity and BMI (all p>0.05). HBV infection was associated with higher alanine transaminase (ALT) and asparate transaminase (AST) levels (both $\mathrm{p}<0.001$ ). No significant differences were found between HBsAg seropositive and seronegative groups in terms of levels of fasting glucose, 2 hour postload glucose and HbAlc and prevalence of diabetes (all $\mathrm{p}>0.05$ ) (table 1 ).

In MIDPC, the median (IQR) of age of HBsAg seropositive group was lower than that in the HBsAg seronegative group (65.0 (58.5-69.5) vs $67.0(61.0-74.0)$ years, $\mathrm{p}<0.001)$. No significant differences were found between the HBsAg seropositive and seronegative groups in terms of the proportions of different occupation, smoking status, alcohol use and physical activity, and levels of BMI (all $\mathrm{p}>0.05$ ). Compared with HBsAg seronegative group, participants with HBsAg seropositivity were younger, had a higher proportion of men and higher education level (all $\mathrm{p}<0.05$ ). Those with HBV infection had higher ALT and AST levels (both $\mathrm{p}<0.001$ ), but similar levels of fasting glucose and prevalence of diabetes (all $\mathrm{p}>0.05$ ) (table 1 ).

Table 2 shows the prevalence of HBsAb seropositivity in GBCS (78.2\%) and MIDPC (65.1\%). In both GBCS and MIDPC, no significant differences in age, sex, occupation, smoking status, alcohol use, physical activity, fasting glucose and prevalence of diabetes were found between HBsAb seropositive and seronegative groups (all $\mathrm{p}>0.05)$. In MIDPC, compared with HBsAb seronegative group, HBsAb seropositive group had higher education and lower BMI (both $\mathrm{p}<0.05$ ). Compared with those with HBsAb seronegativity, participants with HBsAb seropositivity had lower ALT and AST levels (all p<0.001) (table 2).

Table 3 shows that, after adjusting for age, sex, education, occupation, smoking status, alcohol use, physical activity and BMI (additionally adjusting for study sample source while analysing the pooled sample), no significant association between HBsAg serological status and diabetes was found in GBCS (OR=1.12, 95\% CI 0.74 to 1.69), MIDPC (OR=0.83, 95\% CI 0.59 to 1.17 ) or the sample pooled GBCS and MIDPC (OR=0.91, 95\% CI 0.70 to 1.19). After similar adjustment, no significant association between HBsAg serological status and fasting glucose was found in GBCS ( $\beta=-0.01,95 \%$ CI -0.09 to 0.08 ), MIDPC ( $\beta=-0.09,95 \%$ CI -0.39 to 0.20$)$ or the pooled sample $(\beta=-0.03,95 \%$ CI -0.15 to 0.09 ) (table 3 ).

Table 4 shows that, after adjusting for age, sex, education, occupation, smoking status, alcohol use, physical activity and BMI (additionally adjusting for study sample source while analysing the pooled sample), no significant association between HBsAb serological status and 
Table 2 Demographic characteristics and clinical parameters by HBsAb serological status (HBsAb positive vs negative) in GBCS and MIDPC

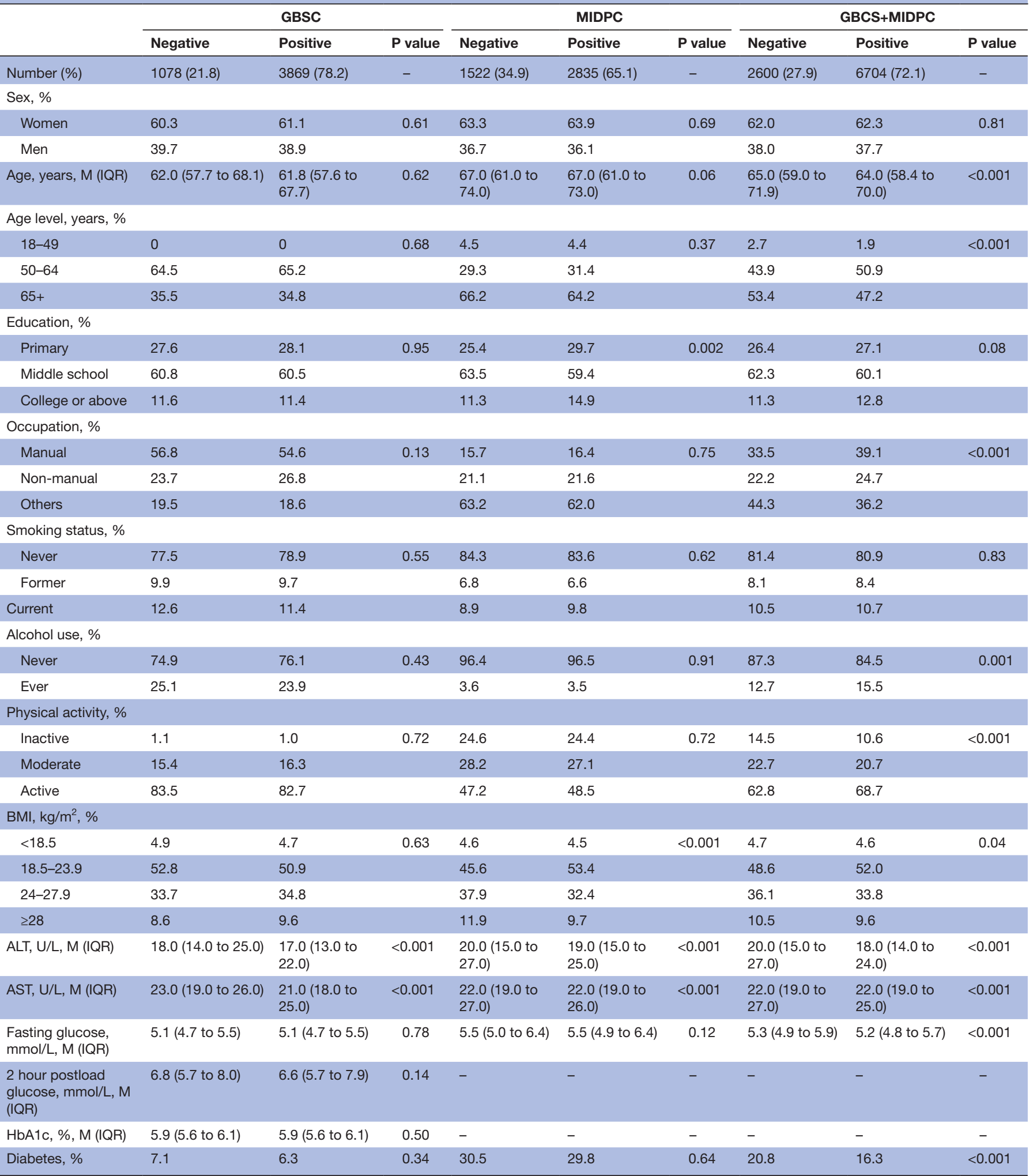

ALT, alanine transaminase; AST, aspartate transaminase; BMI, body mass index; GBCS, Guangzhou Biobank Cohort Study; HbA1c, glycated haemoglobin A1c; HBsAb, hepatitis B surface antibody ; M, median; MIDPC, Major Infectious Disease Prevention and Control .

the presence of diabetes was found in GBCS, MIDPC or the pooled sample (OR $(95 \% \mathrm{CI})$ was $0.85(0.65-1.12)$, $1.00(0.86-1.16)$ and $0.98(0.86-1.12)$, respectively). After similar adjustment, no significant association was found between HBsAb serological status and fasting glucose in GBCS $(\beta=-0.01,95 \%$ CI -0.06 to 0.05$)$, MIDPC $(\beta=0.08$, 
Table 3 Fasting glucose and diabetes status by HBsAg serological status (HBsAg positive vs negative) in GBCS, MIDPC and data with GBCS and MIDPC pooling together

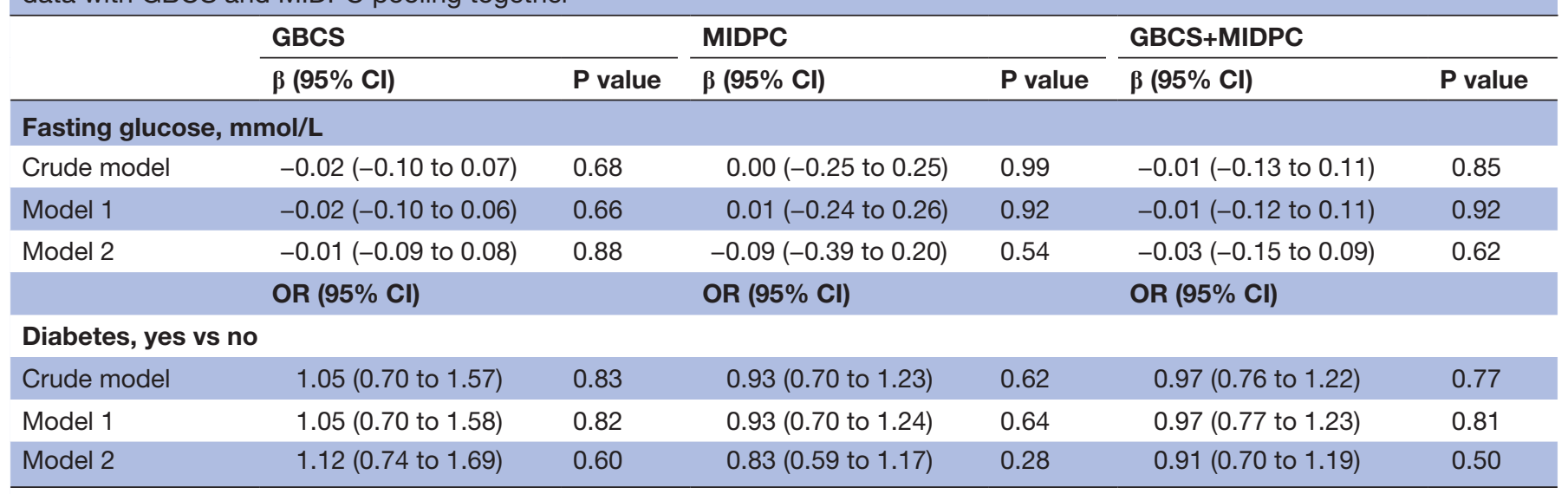

Model 1: adjusting for age and sex.

Model 2: additionally adjusting for education, occupation, smoking status, alcohol use, physical activity, BMI and study sample source, as appropriate.

BMI, body mass index; GBCS, Guangzhou Biobank Cohort Study; HBsAg, hepatitis B surface antigen; MIDPC, Major Infectious Disease Prevention and Control .

$95 \%$ CI -0.06 to 0.21$)$ or the pooled sample ( $\beta=0.04,95 \%$ CI -0.03 to 0.11 ) (table 4 ).

No significant association of serological status of $\mathrm{HBsAg}$ and HBsAb with 2 hour postload glucose and HbAlc was found using data from GBCS. The adjusted $\beta$ was 0.00 , $95 \%$ CI $(-0.24$ to 0.25$)$ and -0.13 ( -0.30 to 0.03$)$ for 2 hour postload glucose, and $-0.02(-0.08$ and 0.05$)$ and -0.02 ( -0.06 to 0.03 ), for HbAlc, respectively (online supplementary table 1). Sensitivity analyses using difference definitions of diabetes in GBCS showed similar null results (online supplementary table 2 and online supplementary table 3 ). Moreover, subgroup analyses by serological status of HBsAg or HBsAb also show no significant association with fasting glucose or diabetes in GBCS or
MIDPC (online supplementary table 4 and online supplementary table 5).

\section{DISCUSSION}

The present study is one of the few studies examining the association of HBsAg and HBsAb seropositivity with diabetes in mainland China. We found no evidence that serological status of HBsAg or HBsAb was associated with the presence of diabetes or glycaemic measures in the two population-based studies. Our findings support results of some previous studies showing that HBsAg seropositivity was not associated with a higher risk of diabetes, ${ }^{16-23}$ and

Table 4 Fasting glucose and diabetes status by HBsAb serological status (HBsAb positive vs negative) in GBCS, MIDPC and data with GBCS and MIDPC pooling together

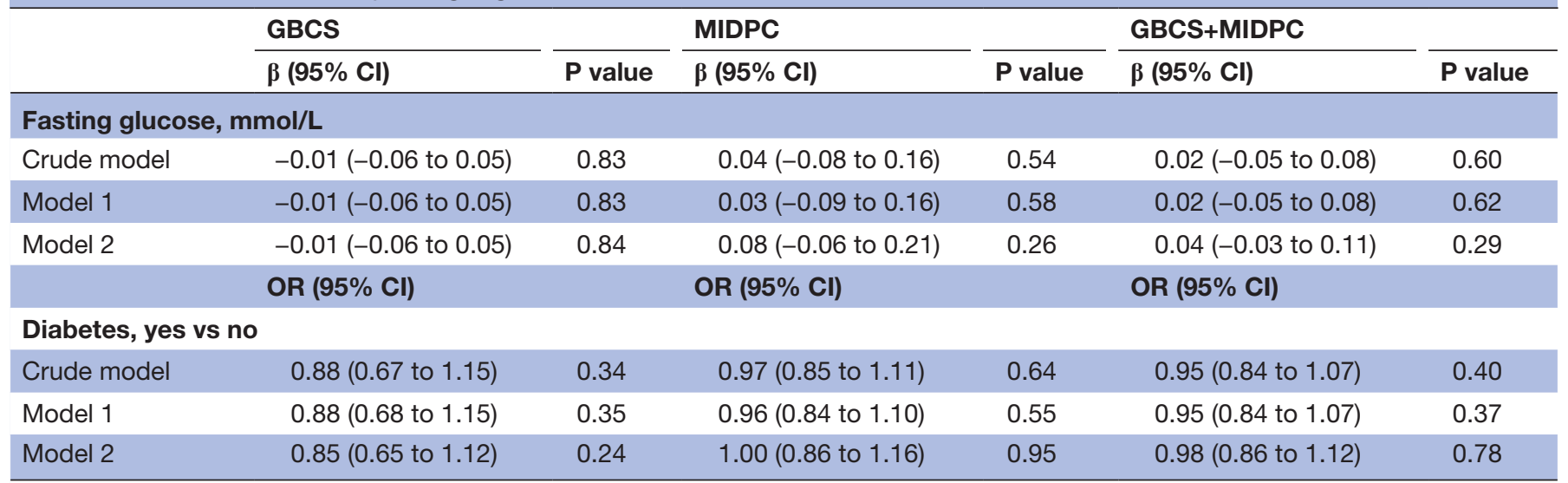

Model 1: adjusting for age and sex.

Model 2: additionally adjusting for education, occupation, smoking status, alcohol use, physical activity, BMI and study sample source, as appropriate.

BMI, body mass index; GBCS, Guangzhou Biobank Cohort Study; HBsAg, hepatitis B surface antigen; MIDPC, Major Infectious Disease Prevention and Control . 
adds to the literature that HBsAb seropositivity was also not associated with diabetes or glycaemic measures.

Our results are consistent with one of the two recent meta-analyses showing no association between HBV infection and diabetes. ${ }^{26}$ In this meta-analysis of 15 observational studies published before 2014 (including seven cohort studies, four case-control studies and four crosssectional studies), no association between HBV infection and diabetes was found after pooling results from four cohort studies and one cross-sectional study $(\mathrm{OR}=0.83$, $95 \%$ CI 0.63 to 1.08$).{ }^{26}$ However, in the other metaanalysis published in 2015, the authors reported a positive association between HBV infection and diabetes based on results pooling eight cross-sectional studies $(\mathrm{OR}=1.40$, 95\% CI 1.04 to 1.90 ) or pooling five case-control studies ( $\mathrm{OR}=1.89,95 \%$ CI 1.08 to 3.30 ), but an inverse association from pooling two cohort studies $(\mathrm{OR}=0.77,95 \%$ CI 0.71 to 0.84$).{ }^{27}$ However, the included studies in the second meta-analysis showed a great heterogeneity $\left(\mathrm{I}^{2}\right.$ value $=93.8 \%, \mathrm{p}<0.001) .{ }^{27}$ Another recent large cohort study of 439708 participants also reported a positive association of HBV infection with the presence of diabetes (OR=1.17, 95\% CI 1.06 to 1.31 ) as well as incident diabetes $(\mathrm{HR}=1.23,95 \%$ CI 1.08 to 1.41$) .{ }^{13}$ Notably, in this cohort study, results of a model without adjustment of fatty liver disease or baseline fasting glucose showed no association between HBV infection and diabetes. ${ }^{13}$ As fatty liver disease is biologically unlikely to cause HBV infection, adjusting for fatty liver disease might lead to an overadjustment bias. ${ }^{28}$ Moreover, the overadjustment bias might also be introduced when adjusting for baseline fasting glucose in the model assessing HBV infection and incident diabetes, ${ }^{13}$ because the diagnosis of incident diabetes included baseline fasting glucose. Adjustment for baseline fasting glucose might thus lead to an overfitting. ${ }^{28}$ In addition, as $80 \%$ of participants in the study were workers from the same company, ${ }^{13}$ generalisability to the general population might have also been limited. Another recent cohort study reported that HBV infection was associated with higher risk of incident diabetes ( $\mathrm{HR}=1.41,95 \%$ CI 1.35 to 1.47 ), but the HBV infection status was defined by medical records rather than serological markers, which underlined more HBV infectionrelated complications such as liver disease rather than simple HBV infection per se. ${ }^{14}$ Due to the controversial results from these previous studies, our finding serves as a complementary evidence, supporting no association between HBV infection and prevalent diabetes.

We found no evidence for a lower risk of diabetes in those with HBsAb seropositivity. HBsAb is a protective antibody serving as a proxy for hepatitis B immunisation. ${ }^{25}$ Results on the association between HBsAb seropositivity and diabetes are scarce. ${ }^{29}{ }^{30} \mathrm{~A}$ study reported negative associations of successful $\mathrm{HBV}$ vaccination (ie, HBsAb seropositive people with a history of HBV vaccination) $(\mathrm{OR}=0.67,95 \% \mathrm{CI} 0.52$ to 0.84$)$ and HBsAb seropositivity ( $\mathrm{OR}=0.75,95 \%$ CI 0.62 to 0.90 ) with diabetes in 15316 participants. ${ }^{29}$ However, HBV vaccination was encouraged after the diagnosis of diabetes, and the study sample had a low prevalence of HBsAb seropositivity $(15.15 \%)$, and successful HBV vaccination rate $(39 \%$ vs $>75 \%$ in the general population after a standard threedose HBV vaccination). ${ }^{31}$ The authors explained that the low rate of successful immunisation could be due to the coexistence or pre-existence of diabetes, because in those with diabetes, a much lower rate of successful immunisation was found, that is, $17 \%$ in diabetic patients versus $41 \%$ in non-diabetic people. ${ }^{29}$ Another cross-sectional study reported that $\mathrm{HBsAb}$ seropositivity was inversely associated with the presence of diabetes in 871 Chinese retired workers from an enterprise $(\mathrm{OR}=0.60,95 \%$ CI 0.39 to 0.92$) \cdot{ }^{30}$ A subgroup analysis by sex showed significant differences in fasting glucose and 2 hour postload glucose levels by serological status of HBsAb in women only. ${ }^{30}$ In southern China where the prevalence of $\mathrm{HBsAb}$ seropositivity as well as diabetes was high, no beneficial effect of HBsAb seropositivity on diabetes was found in our study.

The strengths of our study include large sample size, using data from two population-based studies and accounting for multiple potential confounders. Meanwhile, the present study is one of the few studies which examined the association of HBsAg and HBsAb seropositivity with diabetes in mainland China. While exploring the association between the prevalence of diabetes and HBsAg and HBsAb seropositivity, we have also explored the association of fasting blood glucose with HBsAg and HBsAb seropositivity. However, there are some limitations to our study. First, due to the funding constraints, only $27.3 \%$ of participants in GBCS and 2.6\% in MIDPC had data on both fasting glucose and HBV serological tests and were included in the data analysis. Second, although all residents were invited for free physical examination in the MIDPC, individuals who were more health conscious tended to join and thus volunteer bias might be possible. Our sample was not fully representative of the general population. However, the non-representativeness might not influence internal validity or explain the null associations. Third, our study was a cross-sectional study and thus casual inference could not be confirmed. Further community trials or natural experiments may be useful to confirm the causal relationship between HBV infectious/ immunity and diabetes. Finally, serological status of hepatitis B e-antigen and HBV DNA titre were not measured in GBCS or MIDPC. However, as such measurements are relatively expensive, they were rarely used in populationbased epidemiologic studies.

In conclusion, taking advantage of data from two large cross-sectional studies, we found no association of serological status of HBsAg and HBsAb with the presence of diabetes or glucose measures.

Acknowledgements We acknowledge the contributions from all the authors and we thank all the participants very much for their collaboration.

Contributors YL, LX and YH conceived and designed this study; YL, LX, YH, CJ, WZ, YLJ, TZ and THL contributed to acquisition of data, or analysis and interpretation of 
data; YL, LX, YH, CJ, WZ, YLJ, TZ and THL were involved in drafting the manuscript or revising it critically for important intellectual content. This manuscript is approved by all authors for publication.

Funding This work was funded by the Major Infectious Disease Prevention and Control of the National Science and Technique Major Project (2018ZX10715004), the National Key R\&D Program of China (2017YFC0907100), Natural Science Foundation of Guangdong (2018A030313140), the Natural Science Foundation of China (No. 81941019), the Guangzhou Science and Technology Bureau (201704030132) and the University of Birmingham, UK.

Competing interests None declared.

Patient consent for publication All participants gave written, informed consent before participation for free.

Ethics approval The Guangzhou Biobank Cohort Study was approved by the Guangzhou Medical Ethics Committee of the Chinese Medical Association. The Major Infectious Disease Prevention and Control study was approved by Medical Ethics Committee of School of Public Health, Sun Yat-Sen University.

Provenance and peer review Not commissioned; externally peer reviewed.

Data availability statement Data are available upon reasonable request. Ethical approval in place allows us to share data on request. Please direct such requests to the Data Access Committees of the Guangzhou Biobank Cohort Study (gbcsdata@ hku.hk) and the Major Infectious Disease Prevention and Control (midpc@mail.sysu. edu.cn).

Open access This is an open access article distributed in accordance with the Creative Commons Attribution Non Commercial (CC BY-NC 4.0) license, which permits others to distribute, remix, adapt, build upon this work non-commercially, and license their derivative works on different terms, provided the original work is properly cited, appropriate credit is given, any changes made indicated, and the use is non-commercial. See: http://creativecommons.org/licenses/by-nc/4.0/.

ORCID iD

Yuantao Hao http://orcid.org/0000-0001-8024-5312

\section{REFERENCES}

1 Xia G, Liu C, Cao H, et al. Prevalence of hepatitis B and C virus infections in the general Chinese population. results from a nationwide cross-sectional seroepidemiologic study of hepatitis $A$, $\mathrm{B}, \mathrm{C}, \mathrm{D}$, and $\mathrm{E}$ virus infections in China, 1992. Int Hepatol Commun 1996;5:62-73.

2 Liang X, Bi S, Yang W, et al. Epidemiological serosurvey of hepatitis $B$ in China--declining HBV prevalence due to hepatitis $B$ vaccination. Vaccine 2009;27:6550-7.

3 Zhang WL, Ji ZH, Fu T, et al. [Meta analysis on HBsAg-positive rate among general populations aged 1-59 years, 2007-2016, China]. Zhonghua Liu Xing Bing Xue Za Zhi 2017;38:1278-84.

4 Yang W, Lu J, Weng J, et al. Prevalence of diabetes among men and women in China. N Engl J Med 2010;362:1090-101.

$5 \mathrm{Xu} \mathrm{Y}$, Wang L, He J, et al. Prevalence and control of diabetes in Chinese adults. JAMA 2013;310:948-58.

$6 \mathrm{Hu} \mathrm{H}$, Sawhney M, Shi L, et al. A systematic review of the direct economic burden of type 2 diabetes in China. Diabetes Ther 2015;6:7-16.

7 Zhang $\mathrm{Y}-\mathrm{Y}, \mathrm{Hu} \mathrm{K}-\mathrm{Q}$. Rethinking the pathogenesis of hepatitis $\mathrm{B}$ virus (HBV) infection. J Med Virol 2015;87:1989-99.

8 Xu L, Jiang CQ, Schooling CM, et al. Liver enzymes and incident diabetes in China: a prospective analysis of 10764 participants in the Guangzhou Biobank Cohort Study. J Epidemiol Community Health 2015;69:1040-4

9 Chen SC-C, Tsai SP, Jhao J-Y, et al. Liver fat, hepatic enzymes, alkaline phosphatase and the risk of incident type 2 diabetes: a prospective study of 132,377 adults. Sci Rep 2017;7:4649.
10 Bedarida G, D'Agostino F, Bianchi M, et al. Unexpected lower prevalence of HBsAg in diabetics than in controls. (a study on 2,465 patients). Ric Clin Lab 1982;12:409-15.

11 Jan $\mathrm{C}-\mathrm{F}$, Chen $\mathrm{C}-\mathrm{J}$, Chiu Y-H, et al. A population-based study investigating the association between metabolic syndrome and hepatitis B/C infection (Keelung community-based integrated screening study No. 10). Int J Obes 2006;30:794-9.

12 Li-Ng M, Tropp S, Danoff A, et al. Association between chronic hepatitis $B$ virus infection and diabetes among Asian Americans and Pacific Islanders. Dig Liver Dis 2007;39:549-56.

13 Hong YS, Chang Y, Ryu S, et al. Hepatitis B and $C$ virus infection and diabetes mellitus: a cohort study. Sci Rep 2017;7:4606.

14 Choi HY, Kim Y, Cho H, et al. Risk of diabetes in viral hepatitis $\mathrm{B}$ or $\mathrm{C}$ patients compared to that in noninfected individuals in Korea, 2002-2013: a population-based cohort study. J Viral Hepat 2018;25:272-80.

15 Lu J, Hou X, Tu H, et al. Chronic hepatitis B virus infection status is more prevalent in patients with type 2 diabetes. J Diabetes Investig 2017;8:619-25.

16 Huang Z-S, Huang T-S, Wu T-H, et al. Asymptomatic chronic hepatitis $B$ virus infection does not increase the risk of diabetes mellitus: a ten-year observation. J Gastroenterol Hepatol 2010;25:1420-5.

17 Fraser GM, Harman I, Meller N, et al. Diabetes mellitus is associated with chronic hepatitis $\mathrm{C}$ but not chronic hepatitis B infection. Isr J Med Sci 1996;32:526-30.

18 Wang C-S, Wang S-T, Yao W-J, et al. Community-based study of hepatitis $\mathrm{C}$ virus infection and type 2 diabetes: an association affected by age and hepatitis severity status. Am J Epidemiol 2003;158:1154-60.

19 Spradling PR, Simons B, Narayanan M, et al. Incidence of diabetes mellitus in a population-based cohort of persons with chronic hepatitis B virus infection. J Viral Hepat 2013;20:510-3.

20 Arao M, Murase K, Kusakabe A, et al. Prevalence of diabetes mellitus in Japanese patients infected chronically with hepatitis $C$ virus. $J$ Gastroenterol 2003;38:355-60.

21 Wang C-S, Wang S-T, Yao W-J, et al. Hepatitis C virus infection and the development of type 2 diabetes in a community-based longitudinal study. Am J Epidemiol 2007;166:196-203.

22 Mekonnen D, Gebre-Selassie S, Fantaw S, et al. Prevalence of hepatitis $B$ virus in patients with diabetes mellitus: a comparative cross sectional study at Woldiya General Hospital, Ethiopia. Pan Afr Med J 2014;17:40.

23 Chen H-F, Li C-Y, Chen P, See T, et al. Seroprevalence of hepatitis B and $C$ in type 2 diabetic patients. J Chin Med Assoc 2006;69:146-52.

24 Jiang C, Thomas GN, Lam TH, et al. Cohort profile: the Guangzhou Biobank cohort study, a Guangzhou-Hong Kong-Birmingham collaboration. Int J Epidemiol 2006;35:844-52.

25 Guidelines for the Prevention. Care and treatment of persons with chronic hepatitis B infection, 2015.

26 Zhang J, Shen Y, Cai H, et al. Hepatitis B virus infection status and risk of type 2 diabetes mellitus: a meta-analysis. Hepatol Res 2015;45:1100-9.

27 Cai C, Zeng J, Wu H, et al. Association between hepatitis B virus infection and diabetes mellitus: a meta-analysis. Exp Ther Med 2015;10:693-8.

28 Schisterman EF, Cole SR, Platt RW. Overadjustment bias and unnecessary adjustment in epidemiologic studies. Epidemiology 2009;20:488-95

29 Huang J, Ou H-Y, Lin J, et al. The impact of hepatitis B vaccination status on the risk of diabetes, implicating diabetes risk reduction by successful vaccination. PLoS One 2015;10:e0139730.

30 Li M, Zhou H, Guan Y, et al. Positive hepatitis B surface antibody is associated with reduced risk of diabetes mellitus in retired female Chinese workers. J Diabetes 2016;8:158-61.

31 Mast EE, Weinbaum CM, Fiore AE, et al. A comprehensive immunization strategy to eliminate transmission of hepatitis $B$ virus infection in the United States: recommendations of the Advisory Committee on immunization practices (ACIP) Part II: immunization of adults. MMWR Recomm Rep 2006;55:1-33; quiz CE1-4. 